\title{
Comparative chemical profiling of purified and unpurified Strychnos nux-vomica Linn seeds: An attempt to reduce toxic Brucine content
}

\author{
Research Article
}

\section{Ajay Kumar Meena*1, Poorna Venkataraman², Panda P', Murali Krishna C4, Kusuma Ganji2, Durgesh Dwivedi', Srikanth $\mathbf{N}^{5}$}

1. Regional Ayurveda Research Institute, Aamkho, Gwalior,

2. Captain Srinivasa Murthy Central Ayurveda Research Institute, Chennai.

3. Central Ayurveda Research Institute, Bharatpur, Bhubaneswar.

4. Regional Ayurveda Research Institute, Payakapuram, Vijayawada.

5. Central Council for Research in Ayurvedic Sciences, Ministry of AYUSH, Government of India, New Delhi.

\begin{abstract}
Vishamusthi (Strychnos nux-vomica Linn.), a medicinal plant described as Upavisha (semi-poisonous) group of Ayurvedic Pharmacopoeia of India. Vishamusthi has widely been used and being practiced in several illness namely nervous debility, paralysis, weakness of limbs, sexual weakness, dyspepsia and etc. Ayurveda practices strictly recommend the use of Vishamusthi in therapeutics only after proper shodhana (purificatory procedure) through specific medias such as Gomutra (cow's urine), Godugdha (cow's milk), Goghrita (cow's ghee), and etc. Although various shodhana procedures are recommended in Ayurvedic treatise, but updated scientific researches regarding the shodhana methods are lacking. The present study was undertaken to investigate the physicochemical and phytochemical parameters, quantitative estimation of brucine using cutting edge research tools such as highperformance thin layer chromatography (HPTLC), liquid chromatography-mass spectrometry (LC-MS) and gas chromatography-mass spectrometry (GC-MS) analyses of Vishamusthi seeds before and after purification. The remarkable changes have been observed in different physicochemical parameters, HPTLC, HPLC, GC-MS and LCMS chromatographic profiling before and after shodhana process of Vishamusthi seeds. Quantitative HPLC studies revealed that the process of shodhana resulted in depletion of toxic brucine (chief poisonous constituent of Vishamusthi seeds) reduced to $79.66 \%$ in chloroform extract and $64.54 \%$ in ethanol extract after shodhana process.
\end{abstract}

Key Words: Ayurveda, Shodhana, Brucine, HPLC, Strychnos nux-vomica Linn. Chemical profiling.

\section{Introduction}

Vishamusthi consists of dried seeds of Strychnos nux-vomica Linn. (Family: Fabaceae), a medicinal plant used in Ayurvedic system of medicine for treating various ailments such as nervous debility, paralysis, weakness of limbs, sexual weakness, dyspepsia, dysentery and chronic rheumatism [1]. It is used as a potent rasayana drug for old age problems [2]. Additionally, it is employed in the treatment of anaemia, asthma, bronchitis, colic, intermittent fever, hysteria, etc., in a specific therapeutic dose. Vishamusthi and its alkaloids have been reported to possess anti-oxidant, analgesic, anti-diarrheal, anti-inflammatory, anti-tumor, hepatoprotective and anti-snake venom properties in different experimental models [1]. Although 16 different alkaloids have been isolated and identified from crude

* Corresponding Author:

Ajay Kumar Meena

Assistant Director (Chemistry),

Regional Ayurveda Research Institute,

Aamkho,

Gwalior. India

Email Id: ajaysheera@gmail.com nux vomica, major chemical constituents are strychnine, brucine and their derivatives such as isostrychnine and brucine N-oxide [1]. Strychnine and brucine are the most important and strongly toxic alkaloids not only in the seed but also in the roots, bark, leaves, fruit-pulp, and the hard fruit-shells [3]. Nux-vomica is a highly poisonous at large doses, producing tetanic convulsions and eventually death and in lesser doses it may manifest mental derangement [4]. Illustrations of raw seeds of Vishamusthi and chemical structure of brucine has been provided in Figure 1.

Figure 1. (A) Raw seeds of Vishamusthi, and (B) Chemical structure of brucine
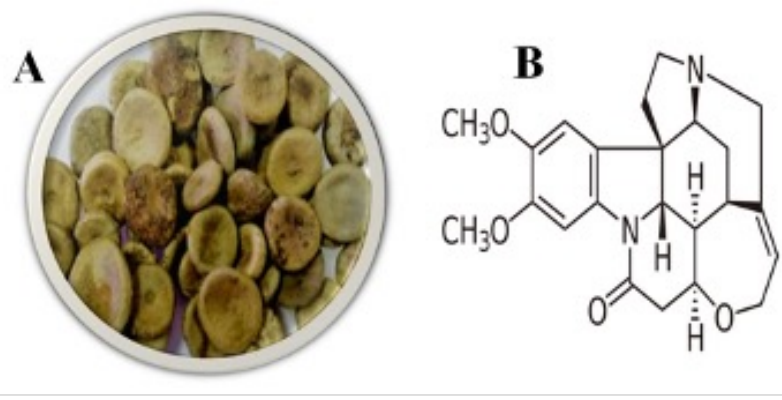
As per the Ayurvedic treatise, Vishamusthi seeds are to be administered in therapeutics only after going through certain purificatory measures (shodhana). In the Ayurvedic literature, it is well established that the 'Visha' (poison) becomes 'Amrita' (nectar) after logical proper purificatory measures in a number of specific media and the ancient physicians of Ayurveda successfully used purified drugs in several diseases [5]. Different methods for specific shodhana procedures are reported for nux vomica seeds [1] [6-9]. In Ayurveda, The shodhana is not only a process of detoxifying toxic substances but also it is a process of enhancing the potency as well as efficacy of the drug [10].Akbar et al. reported the reduction in strychnine content by following the detoxification of nux vomica seeds as mentioned in Unani system of medicine [11]. Mitra et al reported that raw as well as purified vishamusthi exhibited anti-inflammatory activity in formaldehyde induced hind paw oedema in albino rats. An attempt was made by Mitra et al to purify the vishamusthi seeds using cow's urine, cow's milk and cow's urine and cow's milk as media and fund that highest reduction in toxic strychnine and brucine contents after purification in cow's urine for one week followed by boiling in cow's milk for three hours [8]. However, the present study used this method to purify vishamusthi seeds. The present study was undertaken to investigate the physicochemical and phytochemical parameters, quantitative estimation of brucine using cutting edge research tools such as high-performance thin layer chromatography (HPTLC), liquid chromatography-mass spectrometry (LC-MS) and gas chromatography-mass spectrometry (GC-MS) analyses of Vishamusthi seeds before and after purification.

\section{Materials and methods \\ Collection of plant material}

The vishamusthi (Strychnos nux-vomica Linn.) seeds were procured from local crude drug market Chennai, Tamilnadu, India and authenticated at Botany Department of Captain Srinivasa Murthy Central Ayurveda Research Institute, Chennai with the help of flora. Voucher specimen was deposited in the Botany department of the Institute. Copy of authentication certificate is available, if needed.

\section{Shodhana (Purificatory measures) procedure}

The procedure for purification was followed as reported by [8] The seeds of Vishamusthi was cleaned and separated from foreign matter. Seeds of vishamusthi were soaked in Gomutra (Cow's urine) for 7 days and the urine was replaced by fresh Cow's urine every day. Then after, the seeds were cleaned with warm water followed by boiling in Godugdha (Cow's milk) for 3 hours with Dola-yantra method. Then the outer seed coat and embryo were removed from the cotyledons. The seeds were dried in sunlight properly and fried in Cow's ghee on low flame till it gets kapish (slightly brownish) colour, then seeds were pulverized for further studies [12].

\section{HPTLC finger print profile of Visamusthi seeds}

The dried powdered seeds of vishamusthi both materials each approximately $10 \mathrm{~g}$ before and after shodhana process were extracted with $200 \mathrm{ml}$ of absolute ethanol by using soxhlet extraction for $24 \mathrm{hrs}$. The extracts were evaporated to dryness under reduced pressure. The same procedure was followed for chloroform extraction. In order to prepare test solutions, the residues obtained from ethanol and chloroform extracts were weighed and dissolved in methanol using $10 \mathrm{ml}$ volumetric flask, filtered through $0.22 \mu$ membrane filters and used for HPTLC fingerprint profiling and identification of reference standard Brucine biomarker compound. In order to prepare standard curve, $4.1 \mathrm{mg}$ of Brucine was accurately weighed and added to a $10 \mathrm{ml}$ volumetric flask, dissolved in HPLC grade methanol and the volume was made up to $10 \mathrm{ml}$ to obtain $0.41 \mathrm{mg} / \mathrm{ml}$ Brucine stock solution. The solvent system Chloroform: Methanol: Formic acid (8.5:1.5:0.4) was used. In order to run the test, accurately, 151 of the test solution of chloroform and ethanol extracts and 101 of brucine standard solution was applied on different tracks on a precoated silica gel $60 \mathrm{~F}_{254}$ TLC plate (E. Merck) of $0.2 \mathrm{~mm}$ thickness. The plate was developed in the suitable solvent system till the solvent rises to a distance of $8 \mathrm{~cm}$. The plate was observed through TLC Visualizer under UV at $254 \mathrm{~nm}$ and $366 \mathrm{~nm}$ and photos were documented. Finally, the plate was dipped in vanillinsulphuric acid reagent and heated in hot air oven at $105^{\circ} \mathrm{C}$ until the colour of the spots were appeared and photo was documentation under white light and calculate the $R_{f}$ value. Before derivatization the plate was scanned under UV at $254 \mathrm{~nm}$ and $366 \mathrm{~nm}$ using deuterium and mercury lamps respectively. After derivatization plate was scanned at $540 \mathrm{~nm}$ using tungsten lamp. The $\mathrm{R}_{\mathrm{f}}$ values and fingerprint data were recorded by WIN CATS software [13-14].

\section{HPLC chromatographic profiling of Vishamusthi seeds}

In order to prepare test solutions, the residues obtained from chloroform and ethanol extracts of before (unprocessed) and after (processed) shodhana process samples were accurately weighed same amount and dissolved in methanol using $5 \mathrm{ml}$ volumetric flask, filtered through $0.22 \mu$ membrane filters and used for HPLC analysis chromatographic profiling. Chloroform and ethanol extracts of the vishamusthi seeds before and after shodhana process, the processed and unprocessed samples are compared under the same chromatographic conditions. HPLC column of ZORBAX Eclipse XBDC18 $(4.6 \mathrm{~mm} \times 150 \mathrm{~mm}$ ) (particle size $5 \mu \mathrm{m}$ ) was used. Phosphate Buffer and Acetonitrile in the ratio of 70:30 was used as mobile phase. For detection DAD detector at $230 \mathrm{~nm}$ was used. The injection volume of $10 \mu 1$ from processed and purified samples was used. The flow Rate was kept at $1.0 \mathrm{ml} / \mathrm{min}$ [15-16]. 
Quantitative Estimation of Brucine in Vishamusthi seeds by HPLC

The test and standard solutions were prepared as described above. HPLC column of ZORBAX Eclipse XBD- C18 (4.6 mm x $150 \mathrm{~mm})$ (particle size $5 \mu \mathrm{m})$ was used. Phosphate Buffer and methanol in the ratio of 95:5 was used as starting mobile phase. The HPLC operated at gradient elution. For detection VWD Detector at $230 \mathrm{~nm}$ was used. The injection volume of $10 \mu 1$ from processed and purified samples was used. The flow Rate was kept at $1.0 \mathrm{ml} / \mathrm{min}$. In order to prepare calibration curve, $0.41 \mathrm{mg} / \mathrm{ml}$ Brucine stock solution was appropriately diluted further to get a concentration of $0.200,0.100,0.050$ and $0.025 \mathrm{mg} / \mathrm{ml}$ of brucine standards. Each of the standard solution was run through the HPLC and recorded the respective peak areas. In order to estimation of brucine, accurately $10 \mu \mathrm{l}$ of each test solution injected to HPLC system. Record the chromatogram and determine the area of the peak of the test solution corresponding to that of brucine as described above from the calibration curve [16-17].

\section{GC-MS Chromatographic profiling of vishamusthi seeds}

The test solution was prepared by dissolving the dried extract of chloroform in chloroform and methanol solvents of desired volume. Test solutions were filtered and GC-MS analysis was performed as per the standard protocol reported [18].

\section{LC-MS Chromatographic profiling of vishamusthi seeds}

The test solution was prepared by dissolving of ethanol extracts of Visamusthi seeds before and after shodhana process in HPLC grade methanol upto $1.0 \mathrm{ml}$ volume. Test solutions were filtered and LC-MS analysis was performed as per the standard protocol reported [17].

\section{Results}

Preliminary phytochemical screening results showed the presence or absence of certain phytochemicals in the vishamusthi seeds. The comparative preliminary phytochemicals screening results of vishamusthi seeds before and after shodhana a process are tabulated in Table 1.

Table 1. Preliminary phytochemicals screening of Strychnos nux-vomica seeds before and after shodhana process

\begin{tabular}{|c|c|c|c|}
\hline \multirow[b]{2}{*}{ S. No. } & \multirow[b]{2}{*}{ Parameters } & \multicolumn{2}{|c|}{ Strychnos nux-vomica } \\
\hline & & $\begin{array}{l}\text { Before } \\
\text { shodhana }\end{array}$ & $\begin{array}{c}\text { After } \\
\text { shodhana }\end{array}$ \\
\hline 1 & Acids & - & - \\
\hline 2 & Alkaloids & +++ & + \\
\hline 3 & Coumarins & ++ & + \\
\hline 4 & Flavonoids & + & + \\
\hline 5 & Glycosides & + & ++ \\
\hline 6 & Phenols & - & - \\
\hline 7 & Proteins & + & + \\
\hline 8 & Saponins & + & - \\
\hline 9 & Steroids & ++ & + \\
\hline 10 & Sugar & - & - \\
\hline 11 & Tannins & +++ & + \\
\hline \multicolumn{4}{|c|}{$\begin{array}{l}\text { ascertain the quality of the raw material used in th } \\
\text { study. The comparative analysis results } \\
\text { physicochemical parameters for vishamusthi sample } \\
\text { before and after shodhana processes are tabulated } \\
\text { table } 2 \text {. The results of all the parameters complyin } \\
\text { with the Ayurvedic Pharmacopeia of India (AP } \\
\text { standards. The percentage of alcohol soluble extractive } \\
\text { ash content, acid-insoluble ash and pH were foun } \\
\text { reduced and water-soluble extractive, loss on drying } \\
105^{\circ} \mathrm{C} \text { increased in the shodhit (processed) vishamust } \\
\text { seeds as compare to ashodhit ( unprocessed) sample. }\end{array}$} \\
\hline \multicolumn{4}{|c|}{$\begin{array}{l}\text { c-vomica seeds before and after shodhana process } \\
\text { Strychnos nux-vomica }\end{array}$} \\
\hline \multicolumn{2}{|c|}{ Before shodhana } & \multicolumn{2}{|c|}{ After shodhana } \\
\hline \multicolumn{2}{|c|}{6.31} & \multicolumn{2}{|c|}{5.26} \\
\hline \multicolumn{2}{|c|}{7.63} & \multicolumn{2}{|c|}{8.18} \\
\hline \multicolumn{2}{|c|}{12.24} & \multicolumn{2}{|c|}{29.59} \\
\hline \multicolumn{2}{|c|}{5.43} & \multicolumn{2}{|c|}{4.66} \\
\hline \multicolumn{2}{|c|}{1.42} & \multicolumn{2}{|c|}{1.36} \\
\hline \multicolumn{2}{|c|}{0.19} & \multicolumn{2}{|c|}{0.06} \\
\hline
\end{tabular}

HPTLC finger print analysis of vishamusthi seeds

The obtained residue weights for the ethanol and chloroform extractions given in the table 3 .

Table: 3. Extractive values of Strychnos nux-vomica seeds before and after shodhana process

\begin{tabular}{|c|c|c|c|c|c|c|c|c|}
\hline \multirow{2}{*}{ S. No. } & Name of extracts & \multicolumn{2}{|c|}{ Weight of sample (g) } & \multicolumn{2}{|c|}{ Weight of extract (g) } & \multicolumn{2}{c|}{ Extractive value \% $(\mathbf{w} / \mathbf{w})$} \\
\cline { 3 - 8 } & & Before & After & Before & After & Before & 3.1698 \\
\hline 1 & Chloroform extract & 10.1555 & 10.0362 & 0.3219 & 0.4379 & 3.9750 \\
\hline 2 & Ethanol extract & 10.0337 & 10.1131 & 0.3418 & 0.4020 & 3.4065 & 4.3632 \\
\hline
\end{tabular}

A band $254 \mathrm{~nm}$ (Green, $\mathrm{R}_{\mathrm{f}} 0.59$ ) corresponding to brucine is visible in both the reference standard and test solution tracks of chloroform and ethanol extracts of visamusthi seeds before and after shodhana process. HPTLC fingerprint profile of vishamusthi seeds chloroform \& ethanol extracts before and after shodhana process at UV 254 $\mathrm{nm}, 366 \mathrm{~nm}$ and $540 \mathrm{~nm}$ showed in Figure 2-5. Rf values of vishamusthi seeds chloroform \& ethanol extracts before and after shodhana process and brucine reference standard at UV $254 \mathrm{~nm}, 366 \mathrm{~nm}$ and $540 \mathrm{~nm}$ showed in Table 4-6. 
Ajay Kumar Meena et.al., Comparative Chemical profiling of purified and unpurified Strychnos nux-vomica Linn seeds

Table 4. $\mathbf{R}_{\mathrm{f}}$ values of Strychnos nux-vomica seeds chloroform \& ethanol extracts before and after shodhana process and brucine reference standard at UV $254 \mathrm{~nm}$

\begin{tabular}{|c|c|c|c|c|c|c|}
\hline \multirow{2}{*}{ Tracks } & \multicolumn{3}{|c|}{ Before shodhana } & \multicolumn{3}{|c|}{ After shodhana } \\
\hline & Peak & $\mathbf{R}_{\mathbf{f}}$ & Color & Peak & $\operatorname{Max} R_{f}$ & Color \\
\hline \multirow{8}{*}{$\begin{array}{c}\text { Track: } 1 \& 5 \\
\text { Chloroform } \\
\text { extract }\end{array}$} & 1 & 0.22 & Green & - & - & - \\
\hline & 2 & 0.25 & Green & 1 & 0.24 & Green \\
\hline & 3 & 0.28 & Green & 2 & 0.27 & Green \\
\hline & - & - & - & 3 & 0.55 & Green \\
\hline & 4 & 0.59 & Dark green & 4 & 0.59 & Dark green \\
\hline & 5 & 0.62 & Dark green & 5 & 0.67 & Green \\
\hline & 6 & 0.69 & Green & 6 & 0.84 & Green \\
\hline & 7 & 0.93 & Green & & & \\
\hline \multirow{17}{*}{$\begin{array}{c}\text { Track: } 2 \text { \& } 4 \\
\text { Ethanol extract }\end{array}$} & 1 & 0.01 & Green & 1 & 0.01 & Green \\
\hline & 2 & 0.08 & Dark green & 2 & 0.08 & Dark green \\
\hline & - & - & - & 3 & 0.12 & Green \\
\hline & 3 & 0.20 & Green & 4 & 0.17 & Green \\
\hline & 4 & 0.23 & Green & - & - & - \\
\hline & 5 & 0.29 & Green & 5 & 0.28 & Green \\
\hline & 6 & 0.39 & Green & 6 & 0.37 & Green \\
\hline & 7 & 0.45 & Green & - & - & - \\
\hline & 8 & 0.55 & Dark green & 7 & 0.56 & Dark green \\
\hline & 9 & 0.59 & Dark green & 8 & 0.59 & Dark green \\
\hline & 10 & 0.64 & Green & - & - & - \\
\hline & 11 & 0.67 & Green & 9 & 0.67 & Green \\
\hline & 12 & 0.74 & Green & 10 & 0.72 & Green \\
\hline & - & - & - & 11 & 0.78 & Green \\
\hline & 13 & 0.86 & Green & 12 & 0.84 & Green \\
\hline & 14 & 0.91 & Green & 13 & 0.91 & Green \\
\hline & 15 & 0.93 & Green & - & - & - \\
\hline $\begin{array}{c}\text { Track:3 } \\
\text { Brucine } \\
\text { Standard }\end{array}$ & 1 & 0.59 & Dark green & 1 & 0.59 & Dark green \\
\hline
\end{tabular}

Table 5. $\mathrm{R}_{\mathrm{f}}$ values of Strychnos nux-vomica seeds chloroform \& ethanol extracts before and after shodhana process at $\mathrm{UV} 366 \mathrm{~nm}$

\begin{tabular}{|c|c|c|c|c|c|c|}
\hline \multirow{2}{*}{ Tracks } & \multicolumn{3}{|c|}{ Before shodhana } & \multicolumn{3}{|c|}{ After shodhana } \\
\hline & Peak & $\mathbf{R}_{\mathbf{f}}$ & Color & Peak & $\mathbf{R}_{\mathbf{f}}$ & Color \\
\hline \multirow{3}{*}{$\begin{array}{c}\text { Track: } 1 \text { \& } 5 \\
\text { Chloroform } \\
\text { extract }\end{array}$} & 1 & 0.59 & - & 1 & 0.55 & Blue \\
\hline & 2 & 0.93 & Dark blue & 2 & 0.91 & Dark blue \\
\hline & 3 & 0.95 & Dark blue & - & - & - \\
\hline \multirow{6}{*}{$\begin{array}{c}\text { Track: } 2 \text { \& } 4 \\
\text { Ethanol extract }\end{array}$} & 1 & 0.01 & Blue & 1 & 0.01 & Blue \\
\hline & - & - & - & 2 & 0.08 & Dark blue \\
\hline & 2 & 0.56 & Blue & 3 & 0.60 & Blue \\
\hline & - & - & - & 4 & 0.65 & Blue \\
\hline & - & - & - & 5 & 0.71 & Blue \\
\hline & 3 & 0.91 & Blue & 6 & 0.90 & Blue \\
\hline
\end{tabular}

Table 6. $\mathbf{R}_{\mathrm{f}}$ values of Strychnos nux-vomica seeds chloroform \& ethanol extracts before and after shodhana process at 540

\begin{tabular}{|c|c|c|c|c|c|c|}
\hline \multicolumn{7}{|c|}{$\mathbf{n m}$} \\
\hline \multirow{2}{*}{ Tracks } & \multicolumn{3}{|c|}{ Before shodhana } & \multicolumn{3}{|c|}{ After shodhana } \\
\hline & Peak & $\mathbf{R}_{\mathbf{f}}$ & Color & Peak & $\mathbf{R}_{\mathbf{f}}$ & Color \\
\hline \multirow{5}{*}{$\begin{array}{c}\text { Track: } 1 \& 5 \\
\text { Chloroform } \\
\text { extract }\end{array}$} & 1 & 0.53 & Grey & 1 & 0.53 & Grey \\
\hline & - & - & - & 2 & 0.57 & Grey \\
\hline & 2 & 0.68 & Blue & 3 & 0.68 & Grey \\
\hline & 3 & 0.85 & Blue & 4 & 0.86 & Violet \\
\hline & 4 & 0.88 & Violet & - & - & - \\
\hline \multirow{12}{*}{$\begin{array}{c}\text { Track: } 2 \text { \& } 4 \\
\text { Ethanol extract }\end{array}$} & - & - & - & 1 & 0.01 & Grey \\
\hline & 1 & 0.06 & Dark grey & 2 & 0.07 & Grey \\
\hline & 2 & 0.12 & Grey & - & - & - \\
\hline & 3 & 0.19 & Grey & 3 & 0.17 & Grey \\
\hline & 4 & 0.26 & Grey & 4 & 0.31 & Grey \\
\hline & 5 & 0.42 & Grey & - & - & - \\
\hline & 6 & 0.55 & Grey & 5 & 0.53 & Grey \\
\hline & 7 & 0.64 & Grey & - & - & - \\
\hline & 8 & 0.68 & Blue & 6 & 0.67 & Grey \\
\hline & 9 & 0.73 & Grey & - & - & - \\
\hline & 10 & 0.82 & Blue & 7 & 0.83 & Blue \\
\hline & 11 & 0.87 & Violet & 8 & 0.86 & Violet \\
\hline
\end{tabular}


International Journal of Ayurvedic Medicine, Vol 12 (4),807-819

Figure 2. HPTLC fingerprint Profiling of visamusthi seeds chloroform \& ethanol extracts before and after shodhana process and brucine reference standard

At UV 254 nm

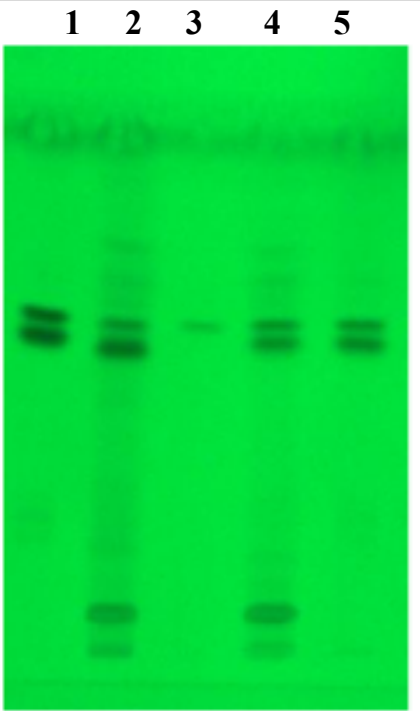

At UV 366 nm

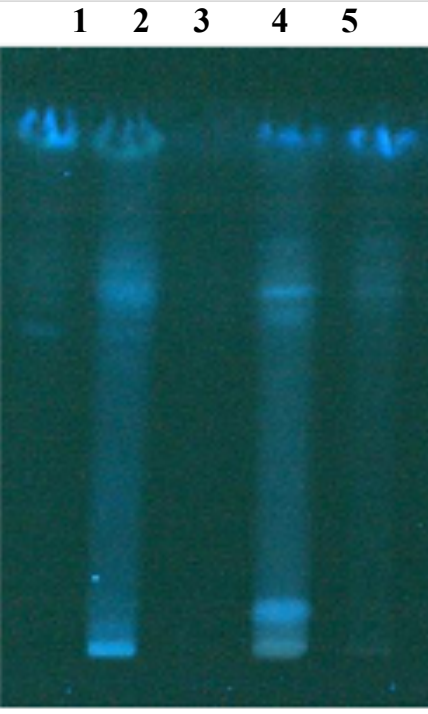

Derivatised with vanillin $\mathrm{H}_{2} \mathrm{SO}_{4}$

Chloroform extract: Track-1: Before shodhana; Track-5: After shodhana;

Reference standard: Track-3: Brucine

Ethanol extract: Track-2: Before shodhana; Track-4: After shodhana

Figure 3. HPTLC fingerprint profile of visamusthi seeds chloroform \& ethanol extracts before and after shodhana process and brucine standard at UV $254 \mathrm{~nm}$

Chloroform extract before shodhana

Chloroform extract after shodhana

Ethanol extract before shodhana

Ethanol extract after shodhana

Brucine reference standard
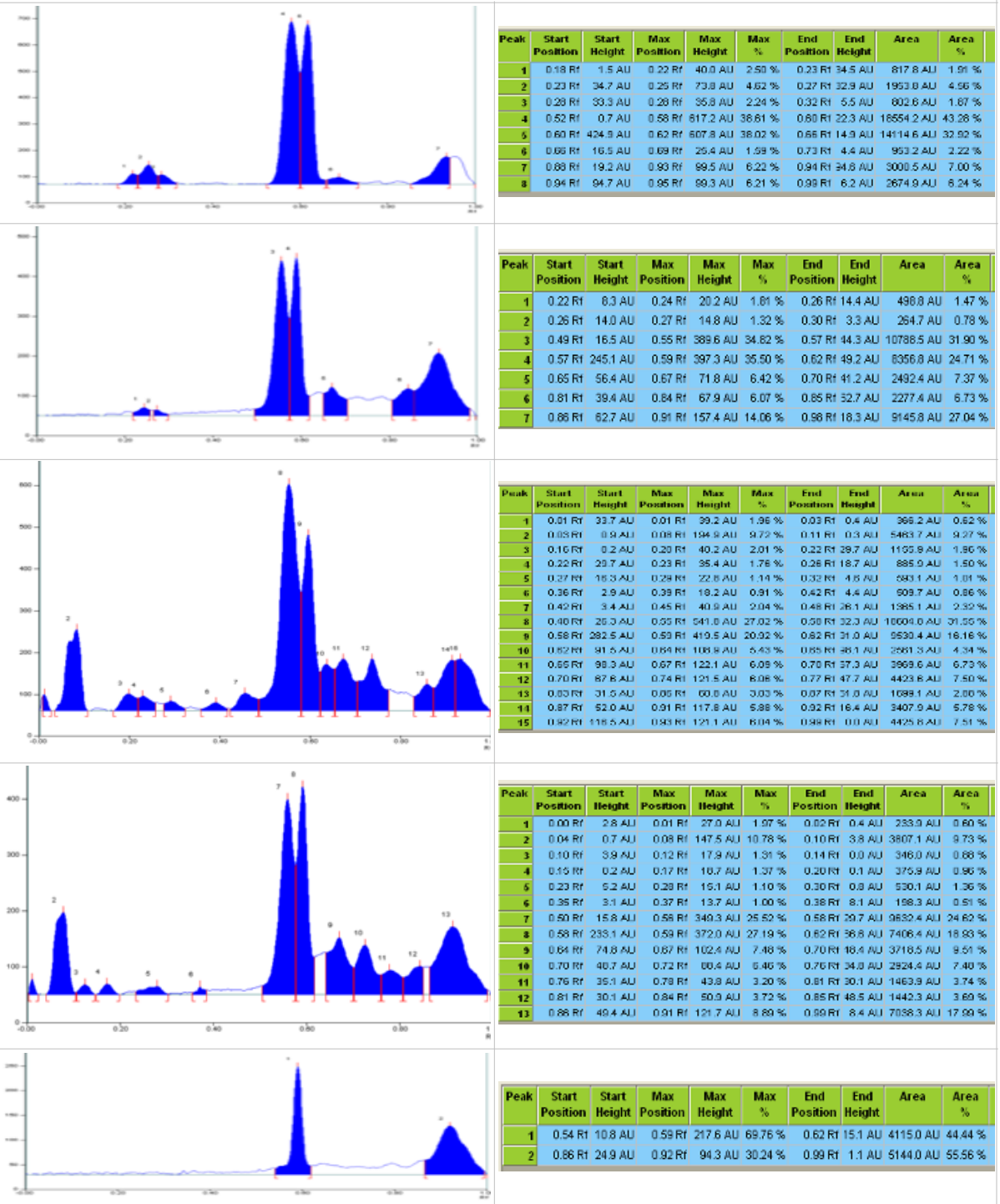
Ajay Kumar Meena et.al., Comparative Chemical profiling of purified and unpurified Strychnos nux-vomica Linn seeds

Figure 4. HPTLC fingerprint profile of visamusthi seeds chloroform \& ethanol extracts before and after shodhana process at UV 366nm

Chloroform extract before shodhana

Chloroform extract after shodhana
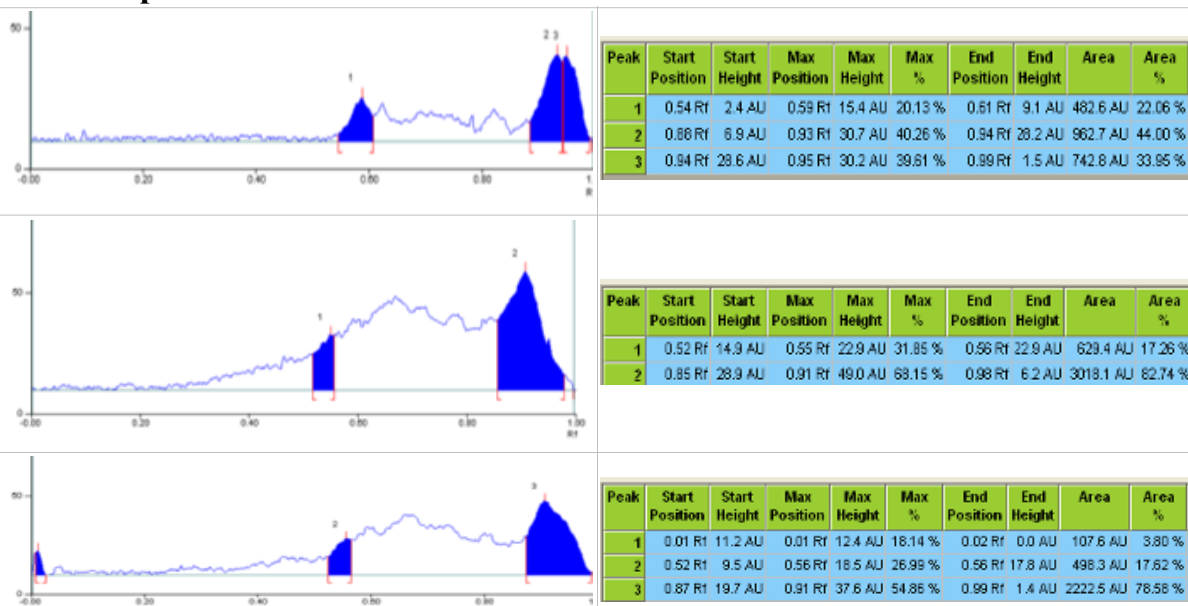

Ethanol extract before shodhana

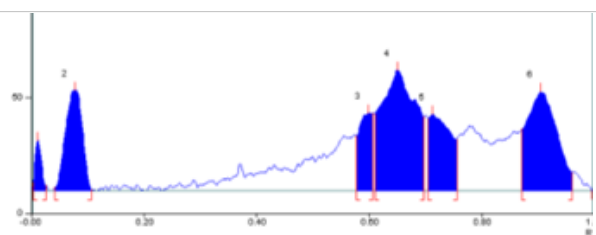

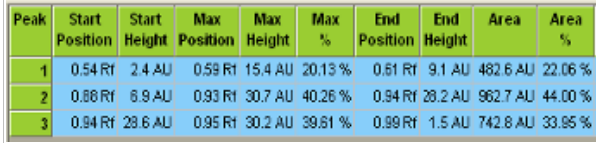

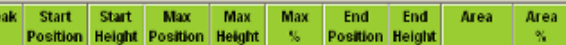

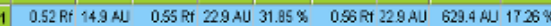
20.85 Rt 28.9 RI 0.91 Rt 490 AU $63.15 \%$ 0.9BRt 62 AU 3013.1 AJ $82.74 \%$
Ethanol extract after shodhana

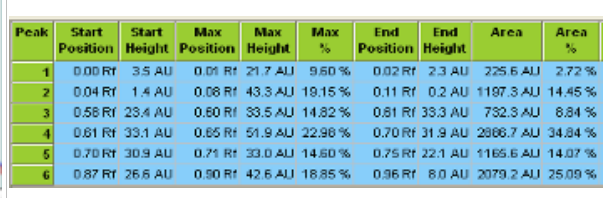

Figure 5. HPTLC fingerprint profile of visamusthi seeds chloroform \& ethanol extracts before and after shodhana process at $540 \mathrm{~nm}$

Chloroform extract before shodhana
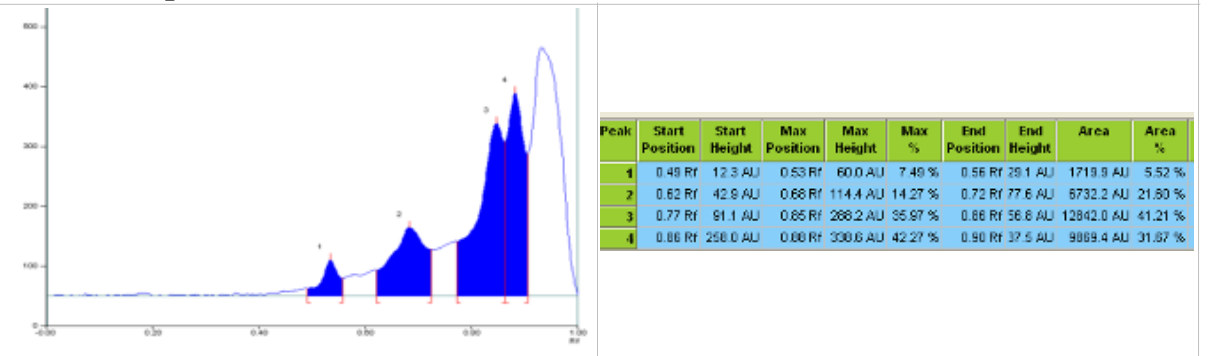

Chloroform extract after shodhana
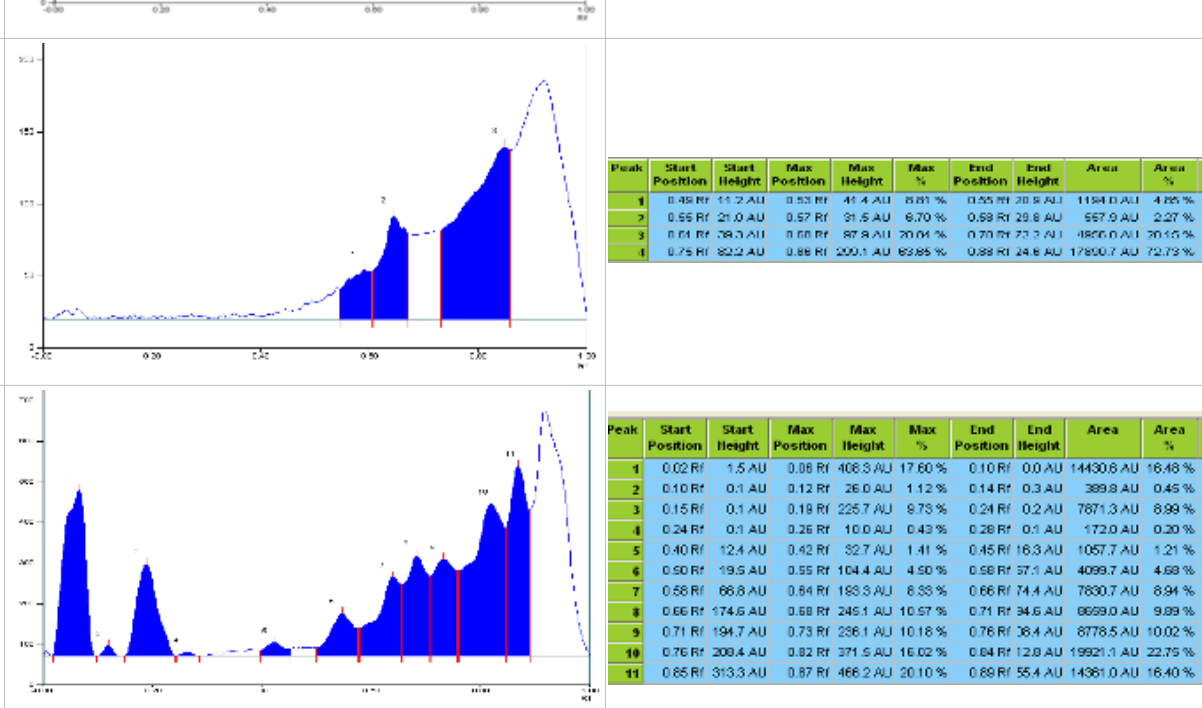

Ethanol extract after shodhana
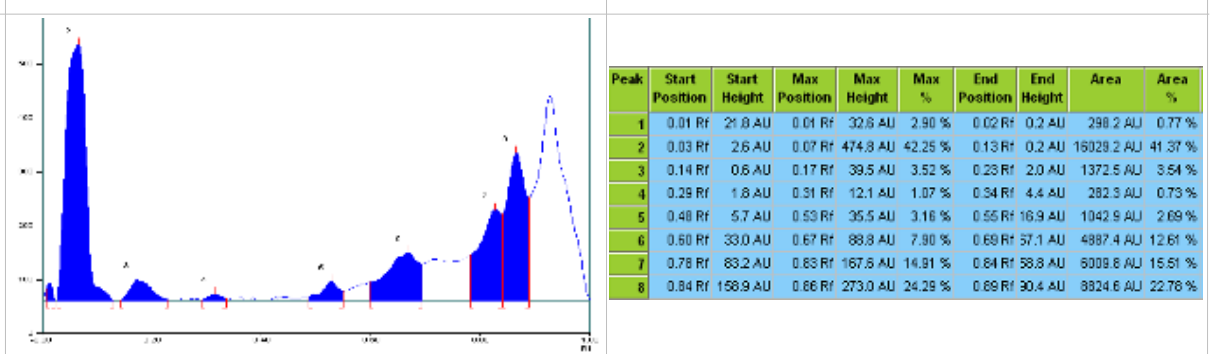


\section{HPLC chromatographic analysis of vishamusthi seeds}

In comparison of HPLC Chromatographic profiling of chloroform extracts, 10 peaks in unprocessed and 08 peaks in processed samples were detected, presented in Figure 6. HPLC Profiling Chromatogram of vishamusthi seeds showed 20 peaks in unprocessed and 6 peaks in processed samples of each ethanol extracts were detected as revealed by Figure 7. It is observed that the peak area of all peaks of processed samples was reduced as compared to unprocessed samples. The results of detailed peak identification and peak area of vishamusthi seeds in chloroform and ethanol extracts before and after shodhana process, are presented in Table 7 and 8 . The remarkable changes have been observed in the chloroform and ethanol extracts HPLC profiling chromatograms of the visamusthi seeds before and after shodhana process.

Table 7. HPLC peak details of Strychnos nux-vomica seeds chloroform extracts before and after shodhana process

\begin{tabular}{|c|c|c|c|c|c|c|c|}
\hline \multicolumn{4}{|c|}{ Unprocessed sample } & \multicolumn{4}{|c|}{ Processed sample } \\
\hline $\begin{array}{c}\text { Peak } \\
\text { No. }\end{array}$ & $\begin{array}{l}\text { Ret. Time } \\
\text { [min] }\end{array}$ & $\begin{array}{c}\text { Area } \\
{\left[\mathbf{m A U} \mathbf{A U}^{*} \mathbf{s}\right]}\end{array}$ & $\begin{array}{c}\text { Area } \\
\%\end{array}$ & $\begin{array}{c}\text { Peak } \\
\text { No. }\end{array}$ & $\begin{array}{l}\text { Ret. Time } \\
\text { [min] }\end{array}$ & $\begin{array}{c}\text { Area } \\
{[\mathbf{m A U} * \mathbf{s}]}\end{array}$ & $\begin{array}{c}\text { Area } \\
\%\end{array}$ \\
\hline 1 & 1.502 & 30.06450 & 0.2583 & 1 & 1.456 & 32.65804 & 0.6177 \\
\hline 2 & 1.622 & 18.43875 & 0.1584 & 2 & 1.626 & 8.16754 & 0.1545 \\
\hline 3 & 1.693 & 13.35016 & 0.1147 & 3 & 1.699 & 8.50321 & 0.1608 \\
\hline 4 & 1.887 & 9.62238 & 0.0827 & 4 & 1.959 & 14.31698 & 0.2708 \\
\hline 5 & 2.001 & 13.45921 & 0.1156 & 5 & 3.939 & 105.70403 & 1.9993 \\
\hline 6 & 2.356 & 13.76966 & 0.1183 & 6 & 11.439 & 597.53699 & 11.3018 \\
\hline 7 & 3.944 & 199.26936 & 1.7122 & 7 & 15.165 & 876.25647 & 16.5734 \\
\hline 8 & 9.736 & 14.43178 & 0.1240 & 8 & 23.248 & 3643.96851 & 68.9217 \\
\hline 9 & 10.281 & 4085.67187 & 35.1056 & - & - & - & - \\
\hline 10 & 13.886 & 7240.17236 & 62.2101 & - & - & - & - \\
\hline Total & $1.16383 \mathrm{E}+04$ & 100.0000 & Total & 5287.11175 & 100.0000 & & \\
\hline
\end{tabular}

Table 8. HPLC peak of Strychnos nux-vomica seeds ethanol extracts before and after shodhana process

\section{Unprocessed sample}

\begin{tabular}{|c|c|c|c|c|c|c|c|}
\hline $\begin{array}{l}\text { Peak } \\
\text { No. }\end{array}$ & $\begin{array}{l}\text { Ret. Time } \\
\text { [min] }\end{array}$ & $\begin{array}{c}\text { Area } \\
{[\mathbf{m A U} \mathbf{A} \mathbf{s}]}\end{array}$ & $\begin{array}{c}\text { Area } \\
\%\end{array}$ & $\begin{array}{c}\text { Peak } \\
\text { No. }\end{array}$ & $\begin{array}{l}\text { Ret. Time } \\
\text { [min] }\end{array}$ & $\begin{array}{c}\text { Area } \\
{\left[\mathrm{mAU}^{*} \mathbf{s}\right]}\end{array}$ & $\begin{array}{c}\text { Area } \\
\%\end{array}$ \\
\hline 1 & 1.495 & 117.95510 & 0.6796 & 1 & 1.489 & 22.73930 & 4.4118 \\
\hline 2 & 1.620 & 1704.65710 & 9.8213 & 2 & 1.591 & 103.70794 & 20.1213 \\
\hline 3 & 1.847 & 101.61682 & 0.5855 & 3 & 1.870 & 12.80527 & 2.4845 \\
\hline 4 & 2.032 & 320.67776 & 1.8476 & 4 & 2.029 & 20.26344 & 3.9315 \\
\hline 5 & 2.602 & 79.79147 & 0.4597 & 5 & 2.760 & 29.12658 & 5.6511 \\
\hline 6 & 2.888 & 449.43628 & 2.5894 & 6 & 4.061 & 326.77200 & 63.3998 \\
\hline 7 & 3.345 & 6267.98975 & 36.1128 & - & - & - & - \\
\hline 8 & 3.701 & 52.62699 & 0.3032 & - & - & - & - \\
\hline 9 & 3.879 & 67.14089 & 0.3868 & - & - & - & - \\
\hline 10 & 3.984 & 72.32104 & 0.4167 & - & - & - & - \\
\hline 11 & 4.126 & 85.54170 & 0.4928 & - & - & - & - \\
\hline 12 & 4.368 & 1400.76794 & 8.0705 & - & - & - & - \\
\hline 13 & 4.578 & 946.41052 & 5.4527 & - & - & - & - \\
\hline 14 & 4.731 & 1320.47620 & 7.6079 & - & - & - & - \\
\hline 15 & 4.836 & 1958.20703 & 11.2821 & - & - & - & - \\
\hline 16 & 5.269 & 1091.63794 & 6.2894 & - & - & - & - \\
\hline 17 & 5.519 & 191.54636 & 1.1036 & - & - & - & - \\
\hline 18 & 5.638 & 199.44115 & 1.1491 & - & - & - & - \\
\hline 19 & 5.775 & 116.65503 & 0.6721 & - & - & - & - \\
\hline 20 & 5.898 & 811.80676 & 4.6772 & - & - & - & - \\
\hline Total & $1.73567 E+04$ & 100.0000 & Total & 515.41453 & 100.0000 & & \\
\hline
\end{tabular}

\section{Processed sample}


Ajay Kumar Meena et.al., Comparative Chemical profiling of purified and unpurified Strychnos nux-vomica Linn seeds

Figure 6. HPLC Profiling Chromatogram of visamusthi seeds chloroform extracts before and after shodhana process Unprocessed sample

Processed sample
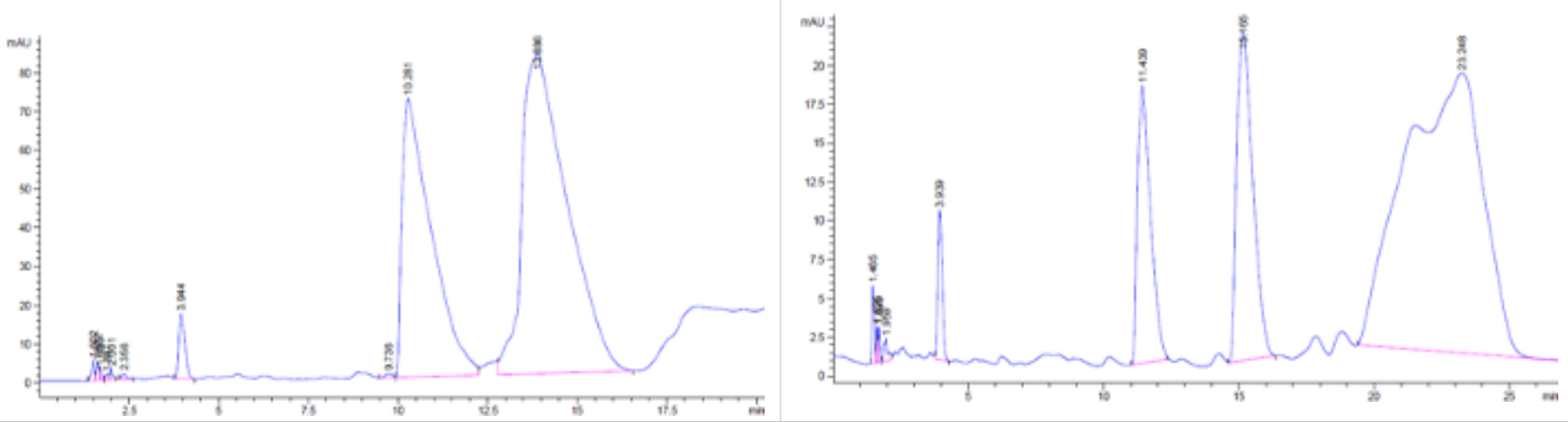

Figure 7. HPLC Profiling Chromatogram of visamusthi seeds ethanol extracts before and after shodhana process Unprocessed sample

Processed sample
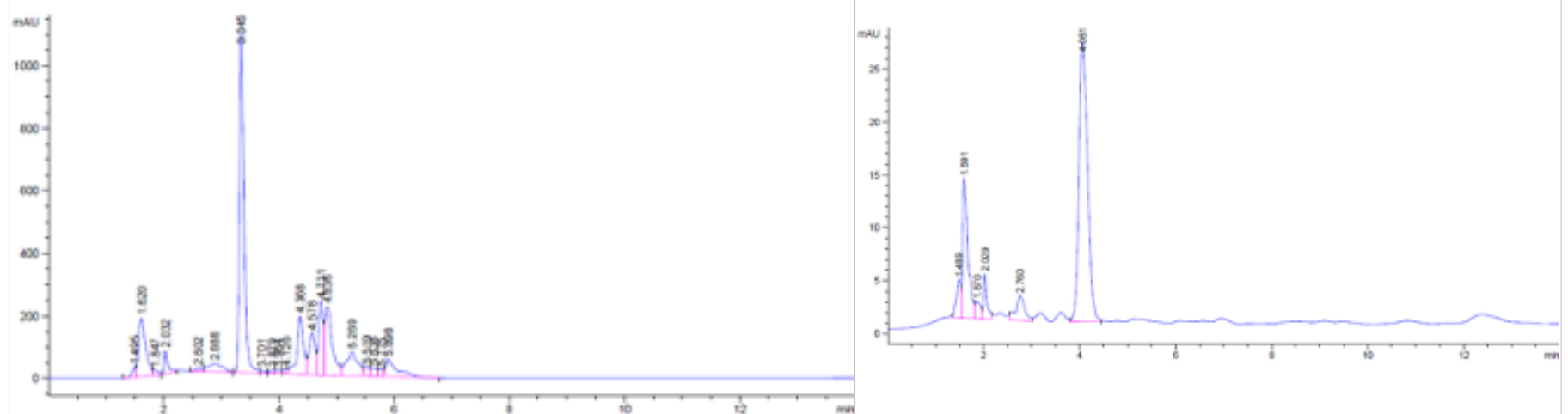

\section{Quantitative analysis of brucine in vishamusthi seeds by HPLC}

Calibration curve was established for peak area Vs concentration of brucine applied, has been shown in figure 8. The amount of brucine present in the residues extracted in ethanol and chloroform for each test sample obtained before and after shodhana samples of vishamusthi seeds, has been provided in table 9 while respected chromatograms has been showing in figure 9. The results obtained from HPLC analysis revealed that the remarkable depletion in the level of brucine after shodhana process of vishamusthi seeds as compared to unprocessed seeds. HPLC analysis showed that the percentage of Brucine was reduced $79.66 \%$ in chloroform extract and $64.54 \%$ in ethanol extract after shodhana process respectively. The HPLC chromatogram of vishamusthi seeds corresponding to standard brucine was showed at a retention time of $6.787 \mathrm{~min}$, at $210 \mathrm{~nm}$ wavelengths.

Figure 8. HPLC Chromatogram of brucine Standard and Calibration curve
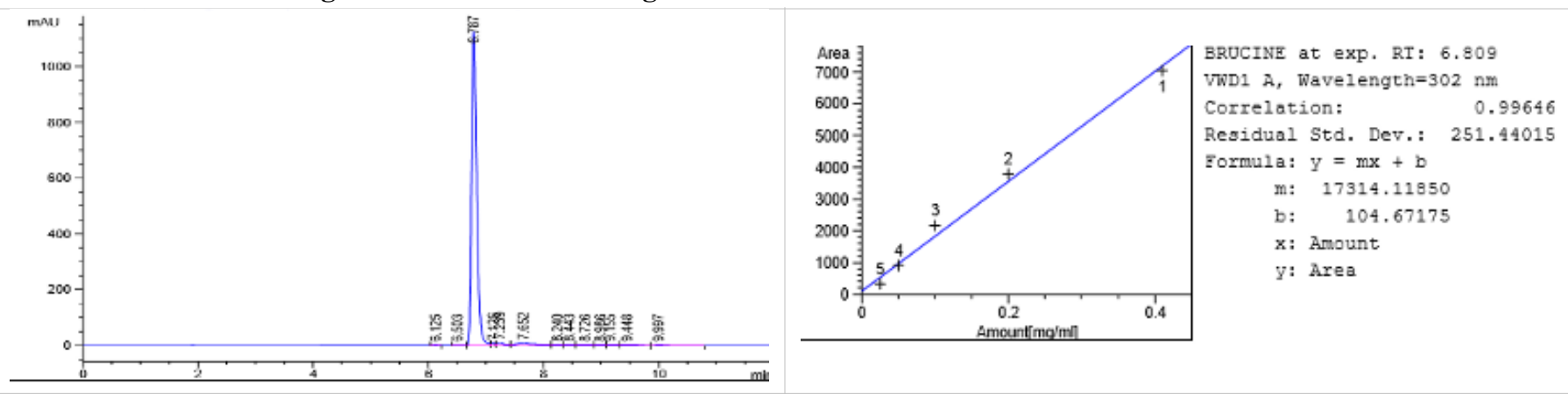

Table 9. Estimation of Brucine in the Strychnos nux-vomica of chloroform and ethanol extracts of before and after shodhana process

\begin{tabular}{|c|c|c|c|c|c|c|}
\hline \multirow{3}{*}{ S. No. } & \multirow{3}{*}{ Name of extracts } & \multicolumn{5}{|c|}{ Brucine $(\% \mathrm{w} / \mathrm{w})$} \\
\hline & & \multicolumn{2}{|c|}{ Before shodhana } & \multicolumn{2}{|c|}{ After shodhana } & \multirow{2}{*}{ Percentage reduced } \\
\hline & & Results & Mean & Results & Mean & \\
\hline \multirow[t]{3}{*}{1} & Chloroform extract & 0.1582 & 0.1567 & 0.0317 & 0.0319 & $79.66 \%$ \\
\hline & & 0.1571 & & 0.0315 & & \\
\hline & & 0.1549 & & 0.0324 & & \\
\hline \multirow[t]{3}{*}{2} & Ethanol extract & 0.0084 & 0.0085 & 0.0030 & 0.0030 & $64.54 \%$ \\
\hline & & 0.0085 & & 0.0030 & & \\
\hline & & 0.0086 & & 0.0030 & & \\
\hline
\end{tabular}


*Percentage of results was given from the means of triplicates for both before and after shodhana samples of chloroform and ethanol extracts.

Figure 9. Estimation of brucine in visamusthi seeds chloroform and ethanol extracts before and after shodhana process

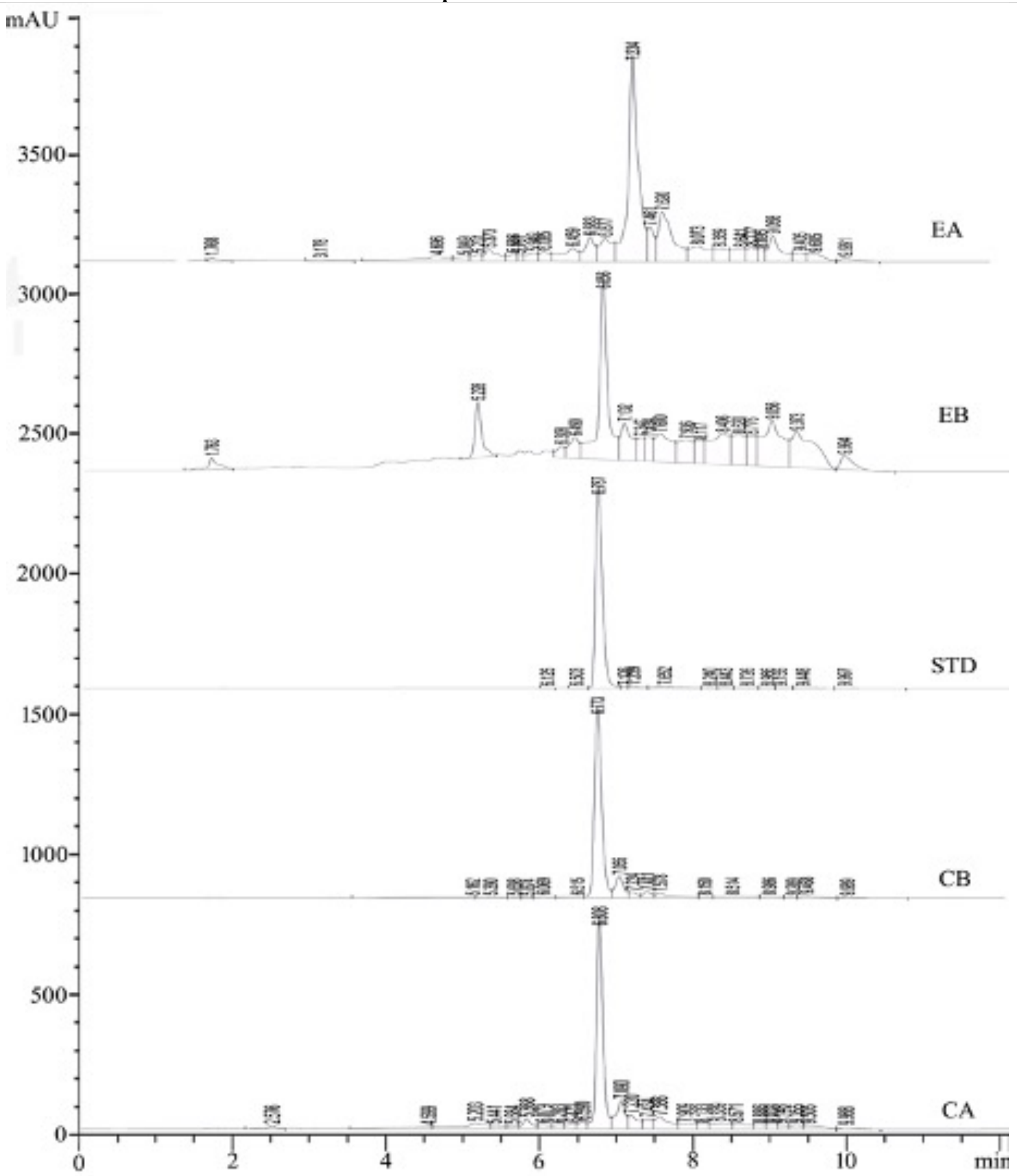

EB:Ethanol extract before shodhana; EA: Ethanol extract after shodhana; STD: Brucine Standard; CB: Chloroform extract before shodhana; CA: Chloroform extract after shodhana

\section{GC-MS Chromatographic analysis of vishamusthi seeds}

The chloroform extracts of the visamusthi seeds before and after shodhana process, the processed and unprocessed samples are compared under the same chromatographic conditions. GC-MS analysis of chloroform extracts of visamusthi seeds shows the presence of 18 peaks in unprocessed sample and 10 peaks in processed sample. The detailed peak identification showed in Figure10 and retention time, peak area, area percentage, compound name and molecular weight are presented in Table 10. The remarkable changes have been observed in the chloroform extracts, GC-MS profiling chromatograms of the Visamusthi seeds before and after shodhana process. 
Ajay Kumar Meena et.al., Comparative Chemical profiling of purified and unpurified Strychnos nux-vomica Linn seeds

Figure 10. GC-MS Chromatogram of visamusthi chloroform extracts before and after shodhana process

Unprocessed sample

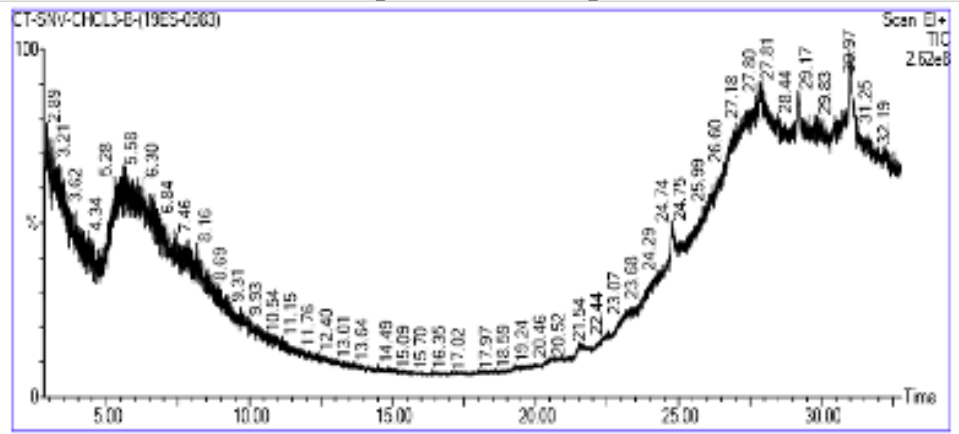

Processed sample

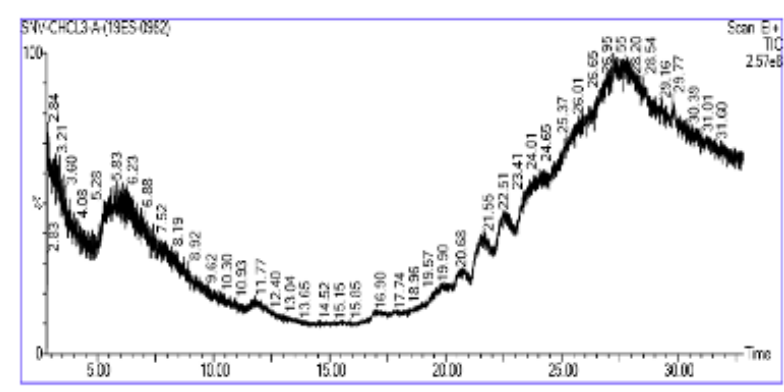

Table 10. GC-MS Peak of Strychnos nux-vomica seeds chloroform extracts before and after shodhana process

\begin{tabular}{|c|c|c|c|c|c|}
\hline \multicolumn{6}{|c|}{ Unprocessed Sample } \\
\hline Peak & RT & Area & Area \% & Name of the compound & Molecular weight \\
\hline 1 & 5.194 & $12,92,763.1$ & 1.689 & \multirow{3}{*}{ Trichloromethane } & 118 \\
\hline 2 & 5.499 & $11,80,258.8$ & 1.542 & & 118 \\
\hline 3 & 6.395 & $10,94,078.6$ & 1.429 & & 118 \\
\hline 4 & 26.818 & $28,64,305.8$ & 3.742 & $\begin{array}{l}\text { 2-Methyl-6-methylene- } \\
\text { octa-1,7-dien-3-ol }\end{array}$ & 152 \\
\hline 5 & 26.873 & $23,38,793.0$ & 3.055 & Oleic acid & 282 \\
\hline 6 & 27.033 & $34,07,232.8$ & 4.451 & 4-Tetradecanol & 214 \\
\hline 7 & 27.123 & $21,27,486.2$ & 2.779 & $\begin{array}{l}\text { Estran-3-one, 17- (acetyloxy) } \\
\text {-2-methyl-, (2.alpha. 5. alpha., 17.beta.)- }\end{array}$ & 332 \\
\hline 8 & 27.323 & $79,17,713.5$ & 10.343 & $\begin{array}{l}\text { Pseduosarsasapogenin-5, } \\
\text { 20-dien methyl ether }\end{array}$ & 428 \\
\hline 9 & 27.459 & $19,63,992.4$ & 2.566 & Hexadecane, 1,16-dichloro & 294 \\
\hline 10 & 27.699 & $1,07,40,507.0$ & 14.030 & Pentanoic acid, 2-(aminooxy)- & 133 \\
\hline 11 & 27.899 & $1,07,29,815.0$ & 14.016 & $\begin{array}{c}\text { 3-Methyl-4-(phenylthio)- 2-prop- 2-enyl-2,5- } \\
\text { dihydrothiophene } \\
\text { 1,1-dioxid }\end{array}$ & 280 \\
\hline 12 & 28.059 & $1,01,48,400.0$ & 13.257 & Pentadecanoic acid & 242 \\
\hline 13 & 28.439 & $14,52,697.0$ & 1.898 & D-Mannitol, 1-O-(22-hydroxydocosyl)- & 506 \\
\hline 14 & 28.534 & $37,58,858.5$ & 4.910 & $\begin{array}{l}\text { Pseduosarsasapogenin- } \\
\text { 5,20-dien }\end{array}$ & 414 \\
\hline 15 & 29.064 & $10,52,095.8$ & 1.374 & D-Mannitol, 1-O-(22-hydroxydocosyl)- & 506 \\
\hline 16 & 29.169 & $45,72,997.5$ & 5.974 & D-Mannitol, 1-decyl sulfonyl & 370 \\
\hline 17 & 30.975 & $87,84,969.0$ & 11.476 & 2,7-Octadiene-1,6-diol, 2,6-dimethyl-, (Z)- & 170 \\
\hline 18 & 31.600 & $11,24,873.8$ & 1.469 & $\begin{array}{l}\text { T-Butyl cyclopentaneperoxy } \\
\text { carboxylate }\end{array}$ & 186 \\
\hline \multicolumn{6}{|c|}{ Processed sample } \\
\hline Peak & RT & Area & Area \% & Name of the compound & Molecular weight \\
\hline 1 & 25.508 & $26,44,144.0$ & 2.315 & $\begin{array}{l}\text { Bicyclo[3.2.1]oct-3-en-2-one, 3,8-dihydroxy -1- } \\
\text { methoxy-7-(7-methoxy-1,3- }\end{array}$ & 388 \\
\hline 2 & 25.713 & $21,82,052.8$ & 1.911 & Z-2-Acetoxy-12- etradecenitrile & 279 \\
\hline 3 & 25.873 & $22,49,412.8$ & 1.970 & Undecanoic acid, 10-bromo & 264 \\
\hline 4 & 26.148 & $34,02,081.0$ & 2.979 & 2,7-Octadiene-1,6-diol, 2,6-dimethyl -, (Z)- & 170 \\
\hline 5 & 27.218 & $4,81,04,500.0$ & 42.122 & Pentanoic acid, 2-(aminooxY)- & 133 \\
\hline 6 & 27.829 & $3,62,07,884.0$ & 31.705 & $\begin{array}{l}\text { Cholesta-8,24-dien-3-ol, } \\
\text { 4-methyl-, (3.beta. 4.alpha.)- }\end{array}$ & 398 \\
\hline 7 & 28.539 & $91,18,143.0$ & 7.984 & $\begin{array}{l}\text { 1-Naphthalene propanol, alpha -ethyldecahydro-5- } \\
\text { (hydroxymethyl)-. }\end{array}$ & 308 \\
\hline 8 & 29.059 & $45,00,372.0$ & 3.941 & Oleic acid & 282 \\
\hline 9 & 29.504 & $18,60,675.4$ & 1.629 & $\begin{array}{l}\text { 2,6-Lutidine 3,5-dichloro-4- } \\
\text { dodecylthion }\end{array}$ & 375 \\
\hline 10 & 29.770 & $39,33,129.0$ & 3.444 & Pregnan-3,11-diol- 20-one & 334 \\
\hline
\end{tabular}

\section{LC-MS Chromatographic analysis of vishamusthi seeds}

The ethanol extracts of the visamusthi seeds before and after shodhana process, the processed and unprocessed samples are compared under the same chromatographic conditions. LC-MS Chromatographic profiling of ethanol extracts of visamusthi seedsshows the presence of 23 peaks in unprocessed sample and 20 peaks in processed sample. The detailed peak identification shown in Figure 11 and retention time, peak area and area percentage are 
given in Table 11. The remarkable changes have been observed in the ethanol extracts LC-MS profiling chromatograms of the visamusthi seeds before and after shodhana process.

Figure 11. LC-MS Chromatogram of visamusthi ethanolic extracts before and after shodhana process Unprocessed sample
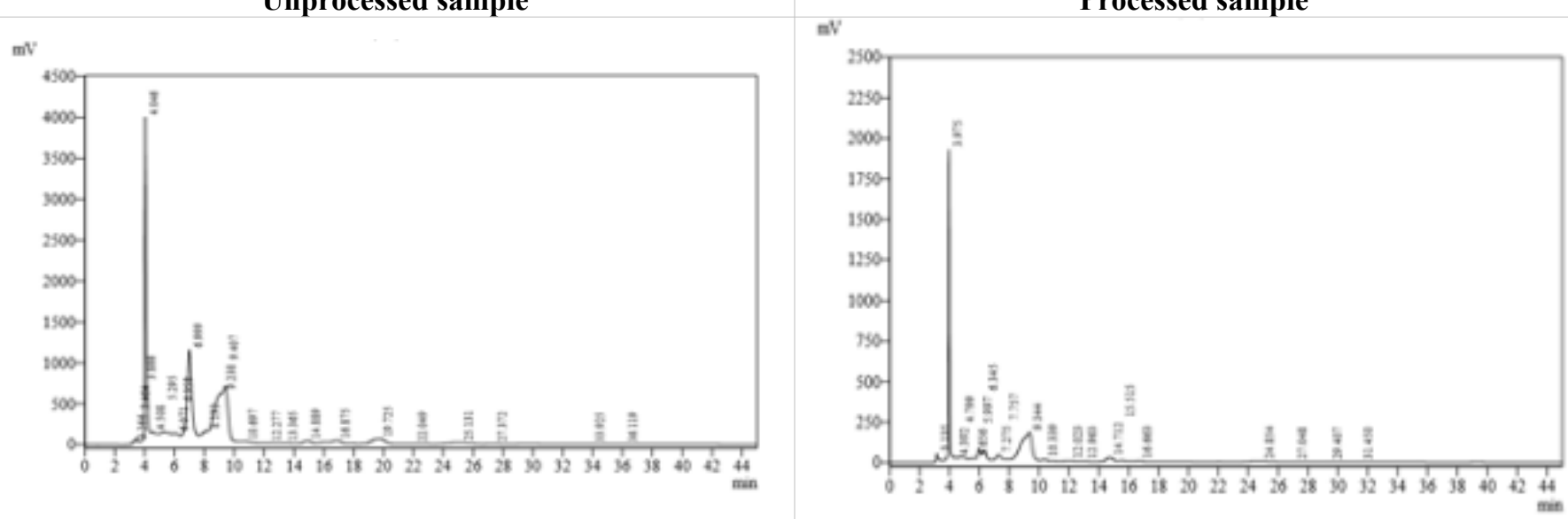

Table 11. LC-MS Peaks details of Strychnos nux-vomica seeds ethanol extracts before and after shodhana process

\begin{tabular}{|c|c|c|c|c|c|c|c|}
\hline \multicolumn{4}{|c|}{ Unprocessed sample } & \multicolumn{4}{|c|}{ Processed sample } \\
\hline Peak & Ret. Time & Area & Area \% & Peak & Ret. Time & Area & Area \% \\
\hline 1 & 3.266 & 385112 & 0.371 & 1 & 3.135 & 474782 & 2.040 \\
\hline 2 & 3.464 & 720513 & 0.695 & 2 & 3.975 & 8737746 & 37.542 \\
\hline 3 & 3.866 & 417639 & 0.403 & 3 & 4.392 & 23029 & 0.099 \\
\hline 4 & 4.046 & 28046821 & 27.055 & 4 & 4.799 & 256553 & 1.102 \\
\hline 5 & 4.508 & 3152079 & 3.041 & 5 & 5.656 & 100787 & 0.433 \\
\hline 6 & 5.295 & 5187641 & 5.004 & 6 & 5.997 & 982434 & 4.221 \\
\hline 7 & 6.071 & 1393362 & 1.344 & 7 & 6.345 & 892718 & 3.836 \\
\hline 8 & 6.358 & 278831 & 0.269 & 8 & 7.275 & 710395 & 3.052 \\
\hline 9 & 6.999 & 22335767 & 21.546 & 9 & 7.757 & 122688 & 0.527 \\
\hline 10 & 8.132 & 254842 & 0.246 & 10 & 9.344 & 8812150 & 37.862 \\
\hline 11 & 9.238 & 18812919 & 18.148 & 11 & 10.339 & 181320 & 0.779 \\
\hline 12 & 9.407 & 13118424 & 12.655 & 12 & 12.023 & 79821 & 0.343 \\
\hline 13 & 10.697 & 261677 & 0.252 & 13 & 12.963 & 16394 & 0.070 \\
\hline 14 & 12.277 & 62142 & 0.060 & 14 & 14.712 & 856245 & 3.679 \\
\hline 15 & 13.365 & 103001 & 0.099 & 15 & 15.515 & 259091 & 1.113 \\
\hline 16 & 14.889 & 1082368 & 1.044 & 16 & 16.663 & 175862 & 0.756 \\
\hline 17 & 16.875 & 2242967 & 2.164 & 17 & 24.854 & 453449 & 1.948 \\
\hline 18 & 19.725 & 3623200 & 3.495 & 18 & 27.048 & 27101 & 0.116 \\
\hline 19 & 22.049 & 131007 & 0.126 & 19 & 29.407 & 92291 & 0.397 \\
\hline 20 & 25.131 & 1746849 & 1.685 & 20 & 31.450 & 19523 & 0.084 \\
\hline 21 & 27.372 & 172334 & 0.166 & - & - & - & - \\
\hline 22 & 33.925 & 32968 & 0.032 & - & - & - & - \\
\hline 23 & 36.119 & 102793 & 0.099 & - & - & - & - \\
\hline Total & 103665258 & 100.000 & Total & 23274378 & 100.000 & & \\
\hline
\end{tabular}

\section{Discussion}

The preliminary phytochemical investigation revealed the presence alkaloids, coumarins, flavonoids, glycosides, proteins, saponins, steroids and tannins. However, there was a decrease in alkaloids, coumarins, steroids and tannins content in processed samples as compared to unprocessed samples. Moreover, there was an increase glycoside content in processed samples as compared to unprocessed samples. The decrease in alkaloidal content, might be an indication of decrease in toxic alkaloidal constituent strychnine and brucine. The water-soluble extractive value in vishamusthi seeds was increased approximately 2.5 folds after in processed samples as compared to unprocessed samples. Whereas, the alcohol soluble extractive value and ash content (also acid insoluble ash) was decreased in processed samples as compared to unprocessed samples. Chloroform as well as ethanolic extractive values (\% w/ w) and weight of extract (g) were increased in processed vishamusthi as compared to unprocessed vishamusthi. Mitra et al reported that, owing to the alkaline nature of cow's urine [pH 8.10 (ranging from $\mathrm{pH} 7.27-8.71)$ ], it facilitates the extraction of alkaloidal content like strychnine as well as brucine from nuxvomica. In HPTLC chromatographic analysis, brucine content was observed at UV spectrum at 254, 366 and $540 \mathrm{~nm}$ with respect to standard brucine $\left(\mathrm{R}_{\mathrm{f}} 0.59\right)$. Cai et al reported that, alkalinity of cow's urine initiated the extraction process and further it was potentiated by milk as boiling in milk converted the strychnine into less toxic isostrychnine [19]. 
HPLC chromatographic analysis revealed a significant decrease in the number of peaks in processed samples as compared to unprocessed samples of both chloroform and ethanolic extracts. There was more than two fold decrease in peaks were observed in the processed samples of ethanolic extract. Quantitative analysis of brucine by HPLC confirmed a significant decrease in brucine content to $79.66 \%$ and $64.54 \%$ in processed samples of chloroform and ethanolic extract, respectively. It has been reported that brucine content in raw/crude (Unpurified/unprocessed) vishamusthi seed is $0.77 \%$. Whereas, brucine content after purification in cow's milk and is reduced to $0.68 \%$. It is worthy to mention that brucine content after sequential purification in cow's urine, milk, ghee is $0.57 \%$ [20].

GC-MS analysis of chloroform extracts of vishamusthi seeds shows the presence of 18 peaks in unprocessed sample and 10 peaks in processed sample. Whereas, LC-MS Chromatographic profiling of ethanol extracts of vishamusthi seeds shows the presence of 23 peaks in unprocessed sample and 20 peaks in processed sample. V. G. S. Sharma \& Reddy reported that acute toxic dose of vishamusthi unprocessed seed ethanolic extract was ranging from $15-50 \mathrm{mg} / \mathrm{kg}$, whereas seeds purified in media (Cow's urine, milk and ghee) showed acute toxic dose ranging from $300-600 \mathrm{mg} / \mathrm{kg}$ [21].

Many Ayurveda formulations containing vishamusthi seeds are used vatadosha (mainly responsible for pain and neurological disorders). Some of them formulations are Karaskara ghritham, Agnitundi rasa, Navajivana rasa, Lakshmivilasa rasa, Sulnirmulana rasa, Supti vatari rasa, Sarameya vishahara yoga, Vishatinduka taila, Krimimudgara rasa, Mahavishagarbha taila, Ekangavira rasa and Vishatinduka vati.

\section{Conclusions}

The percentage of alcohol soluble extractive, ash content, acid-insoluble ash and $\mathrm{pH}$ were found reduced and water-soluble extractive, loss on drying at $105^{\circ} \mathrm{C}$ increased in the shodhit (processed) vishamusthi seeds as compare to ashodhit (unprocessed) sample. The remarkable changes have been observed in different physicochemical parameters, HPTLC, HPLC, GC-MS and LC-MS chromatographic profiling before and after shodhana process of Vishamusthi seeds. In the HPLC chromatographic profiling it is observed that the peak area of all peaks of processed samples was reduced as compared to unprocessed samples.

HPLC studies revealed that the process of shodhana resulted in depletion of more toxic brucine, which is the chief poisonous constituent of vishamusthi seeds. Study showed that the percentage of brucine was reduced $79.66 \%$ in chloroform extract and $64.54 \%$ in ethanol extract after shodhana process respectively.

\section{Acknowledgements}

The authors are very grateful to the DirectorGeneral, CCRAS, Ministry of AYUSH, New Delhi, for providing encouragement and facilities for carrying out this work.

\section{Reference}

1. Mitra S., Kumar V., Ashok B., Acharya R. N. \& Ravishankar B. A comparative anti-inflammatory activity of raw and processed Kupeelu (Strychnos nux-vomica Linn.) seeds on albino rats. Anc. Sci. Life. $2011 ; 31 ; 73-75$.

2. Pandey, G. Anti-Aging Herbal Drugs of India. Delhi Sri Satguru Publ. 248 (2002).

3. Council of Scientific \& Industrial Research. The Wealth of India: A Dictionary of Indian Raw Materials and Industrial Products.[Raw Materials]. Vol. 4 (Council of Scientific and Industrial Research, 1956.

4. CCRAS. Database of Indian Medicinal Plants. Vol. 5. New Delhi Doc. Publ. Div. CCRAS; 2005. p. 139.

5. Sharma R. K. \& Dash B. Charaka samhita-text with English translation and critical exposition based on Chakrapani Datta's Ayurveda dipika. India: Chowkhamba Varanasi. 2003.

6. Trikamji, A. Y. Siddha Yoga Sangraha. Shri Baidhnath Ayurved Bhavan Ltd 84, 2000.

7. Shastri KN; Sharma S. N. Rasa Tarangini. Hindi Comment. Delhi Motilal Banarasi Das. 2004; 15, 59-61.

8. Mitra, S., Shukla, V. J. \& Acharya, R. Effect of purificatory measures through cow's urine and milk on strychnine and brucine content of Kupeelu (Strychnos nuxvomica Linn.) seeds. African J. Tradit. Complement. Altern. Med. AJTCAM , 2012;9; 105-111.

9. Mitra S., Shukla V. J. \& Acharya, R. Effect of Shodhana (processing) on Kupeelu (Strychnos nuxvomica Linn.) with special reference to strychnine and brucine content. AYU. 2011,32, 402-407.

10. Shastri J. L. N. Dravyaguna Vijnana. Vol-1. Varanasi Choukhamba Orient. 2004;382.

11. Akbar, S., Khan, S. A., Masood, A. \& Iqbal, M. Use of Strychnos nux-vomica (Azraqi) seeds in Unani system of medicine: role of detoxification. African J. Tradit. Complement. Altern. Med. 2010;7.

12. The Ayurvedic Formulary of India. The Ayurvedic Formulary of India, Part I, II \& III. (Department of Ayurveda, Yoga \&amp; Naturopathy, Unani, Siddha and Homoeopathy (AYUSH), Ministry of Health and Family welfare, Govt. of India., 2011.

13. Wagner H. \& Bladt S. Plant drug analysis: a thin layer chromatography atlas. Springer Science \& Business Media, 1996.

14. Meena A. K. et al. Determination of Gallic Acid in Ayurvedic Polyherbal Formulation Triphala churna and its ingredients by HPLC and HPTLC. Res. J. Pharm. Technol. 2018;11, 3243-3249.

15. Meena A. K. et al. Estimation of Withaferin-A by HPLC and standardization of the Ashwagandhadi lehyam formulation. Heliyon, 2021;7, e06116.

16. Tang H.-B. et al. HPLC-DAD method for comprehensive quality control of Semen Strychni. Pharm. Biol. 2013; 51, 1378-1383.

17. Hashim A. et al. A reverse phased high-pressure liquid chromatographic method for the estimation 
of a poisonous matter in Strychnos nux-vomica. J. Adv. Pharm. Technol. Res. 2015; 6, 108.

18. Meshram A., Kumar A. \& Srivastava N. Gas chromatography-mass spectrometry (GC-MS) analysis of alkaloids isolated from Epipremnum aureum (Linden and Andre) Bunting. Int J Pharma Sci Res 2015;6; 337-342.

19. Cai B. C., Hattori M. \& Namba T. Processing of nux vomica. II. Changes in alkaloid composition of the seeds of Strychnos nux-vomica on traditional drug-processing. Chem. Pharm. Bull. (Tokyo). 1990;38;1295-1298.
20. Ilanchezhian R., Roshy J. C. \& Acharya R. Importance of media in shodhana (purification / processing) of poisonous herbal drugs. Anc. Sci. Life 30, 54-57 (2010).

21. Sharma V. G. S. \& Reddy M. N. Comparative toxicological evaluations (Acute toxicity) of raw (unprocessed) and processed (detoxified / shodhit) extracts from kupeelu (strychnos nux-vomica Linn.) in wistar rats. Int. J. Pharm. Sci. Rev. Res. 2016;40, 33-35. 